\title{
Applying the Theory of Structuration in Enterprise Architecture Design
}

\author{
Dominic M. Mezzanotte, Sr., Josh Dehlinger and Suranjan Chakraborty \\ Department of Computer and Information Science, Towson University, Towson. Maryland, U.S.A. \\ \{dmezzanotte,jdehlinger, schakraborty\}@towson.edu
}

\begin{abstract}
Enterprise Architecture (EA) defines the infrastructure that acts as a force ensuring alignment of an organization's strategic business plan(s) with its information technology (IT) infrastructure. Current EA frameworks are techno-centric approaches in that business goals, strategies and governance are considered only from the informational aspects of automation. However, EA frameworks do not address the behavioral patterns exhibited by users and the effects of organizational change introduced by EA. Failure to recognize and address negative behavioral patterns often results in the partial or complete abandonment of the EA. This paper extends our approach incorporating ideas from the Theory of Structuration to address human behavior as a significant input to formulating and implementing an EA. The contribution of this paper progresses detailing of human and organizational behavior and utilizing aspects of organizational and behavioral theory to further a framework that focuses on communication as a part of EA development.
\end{abstract}

\section{Introduction}

Enterprise Architecture (EA) is a continually emerging and evolving approach for capturing knowledge about, and documenting, an organization's plan to implement new or modified information technology (IT) [19]. The methodologies used in EA design can range from a very broadly-focused enterprise-wide strategic Information Systems (IS) plan encompassing all aspects of the organization's information and technological needs (the IT infrastructure, hardware, software, and procedures) to one limited in scope and directed at a specific domain and IS application. The product of this effort, an Enterprise Architecture Plan (EAP), expresses an organization's intent and desire to align its strategic business plan with its information technology [14].

The current Enterprise Architecture Framework (EAF) processes rely solely on utilizing the epistemological characteristics, culture and behavioral patterns and policies of the enterprise [9][16]. However, the interactions, goals, objectives, and behavior of stakeholders involved in the development of an EAP are often at odds with the strategic plans of the enterprise resulting in the EAP being only partially implemented or, in many cases, being completely abandoned with many causal factors cited for failure [6][13][17]. Statistics related to EA failure range upwards from $66 \%$ to $82 \%$ [17] in the private sector to over $84 \%$ [4] in public organizations [4][6][17].
Explaining each of the factors associated with failure is often complex and difficult. The complexity arises from the interactions of technology and those tools (software, procedures and processes) required and used to transform collected data into information [4][9][16]. In addition the difficulty in teasing out the individual factors lies in the complex dynamics underlying how stakeholders interact with and react to the technology plan intrinsic to the EA design and implementation.

Formulating and implementing EA represents a significant change in the organizational structure and frequently meets with resistance from stakeholders reluctant to change. Resistance to change is not atypical human behavior. This behavior is often characterized by resultant changes in organizational behavior, usually attributed to management [1][7][11][12], while individuals and organizations historically trend towards a stable environment and a state of equilibrium [9]. In some cases, individual, and consequently organizational, resistance to change may result in work intentionally being sabotaged either overtly or covertly thus allowing the organization to revert to the known equilibrium of the past [11][12].

Where technology is involved, user behavior often reveals itself in the way in which the technology is presented. For example, if the technology is introduced unexpewctedly and without any input from or concern about stakeholders, it then may be either accepted or rejected by those involved in the technological transition [4][9][11][12][16]. When change is unexpected, user resistance to the technology can be considerable [9]. We can conclude that the manner in which the EA design effort is supported and deployed by a development methodology (in the case of EA an EAF), as well as the design and implementation approach taken by organizational management significantly affects how stakeholders perceive and behave during the EA design process [1][7]. Consequently, user behavior should be viewed as the result of, and due to cognitive processes learned resulting from experiencial organizational behavior and their ability to adapt to a changing environment which would in turn affect their respective future capacity for handling and accepting change [1].

The behavior displayed is often in defensive routines and mechanisms such as stakeholder attempts to deceive, manipulate, or distort information during the EA design 
process [11][12]. Therefore, user behavior becomes a critical and potentially costly component to EA affecting project success and/or failure with a need to mitigate stakeholder and organizational resistance to change and their respectibe attitudes towards new technology[16].

In the EAF processes used in the major EA methodologies (Zachman Architectural Framework [20], ToGAF [15], FEAF [2], and DoDAF) [1]), the view of user and organizational behavior varies from being either cursorily considered or completely avoided in the EAP process [18][19]. Yet, any discourse regarding technology should be inextricably linked to the social context of its formulation and use. In this context, technology, if taken singularly and in isolation, can be easily constrained resulting in limited stakeholder innovation, creativity, participation, interest, and commitment when designing and implementing the new EA procedures, tasks, techniques, tools, user knowledge requirements, roles, and responsibilities without considering their impact on human behavior [7][11][12][14].

This paper considers both user and organizational behavior as multi-faceted, complex systems/entities that together recursively affect the design, implementation and use of enterprise-wide information technology. The emphasis in this paper explores the benefits of using a socio-communicative based framework that supplements and supports the recursive nature of stakeholder behavior for formulating and designing EA in organizations that builds on earlier work [13].

The remainder of this paper is as follows. Section 2 reviews several EAF methodologies to ascertain how they cope, or fail to cope, with the human and organizational behavioral complexities associated with deployment of enterprise-wide technology exhibited during EA design. Section 3 discusses the Theory of Structuration [7] and its potential for application to IT [16] and EA design with a lens to mitigating EA failure. Section 4 briefly describes the preliminary processes of a framework that takes into account user and organizational behavior enhancing existing architectural framework methodologies or its use as a framework by itself. Finally, Section 5 provides a discussion, some concluding remarks, and future research towards an enhanced approach to EA.

\section{An Analysis of Current Enterprise Architecture Frameworks and Stakeholder Behavior}

In the context of this paper, Project Management (PM) focuses on developing "working software" [5] while EA and the Enterprise Information Architect (EIA) focuses on planning and describing the overall strategic technological solutions and infrastructure which map to the current and future operating model of the enterprise [9][14]. The scope of the EIA's work, therefore, must be wider and include skills related to business acumen as well as sociological and psychological soft people skills to be effective. Confusing the two activities almost always results in misdirection of effort and a reinforcement of misunderstanding on the part of stakeholders leading to EA failure.

Over the past twenty plus years, several EAF models have been developed and deployed. Included in the following list are five popular EAF methodologies:

- Zachman Enterprise Architecture (Z|FA) [20]

- The Open Group Architecture Framework (TOGAF) [15]

- The Federal Enterprise Architecture Framework (FEAF) [2]

- The Department of Defense Architecture Framework (DoDAF) [1]

The EAF approach taken by each of these unique methodologies provides a framework, a set of tools, used to document and develop a broad range of EA technologyoriented alternatives. The respective collective strengths of each framework lies in their paradigm for documenting the specifications and requirements for IT applications while defining an EAP (an EA blueprint) consisting of and describing a common EA vocabulary, the methods, disciplines, processes, and system and subsystem relationships that communicate the architecture of the EA [20][14][15]. The frameworks impose a disciplined, comprehensive technocentric regimen of methods, principles, and practices to be used by the EIA resulting in an EAP that allows the organization to then focus on how best to use and align technology with the organization's strategic business plan.

The weakness of each EAF is that the concentration of work is technically oriented and aimed primarily at producing a set of deliverables focused on the technical aspects of EA design specifications, potential modeling schemes, and implementation of the design. The EAP documents the organization's structure, business processes, Information Systems (IS), and related IT infrastructure at a technical, domain-level architecture that fails to take into consideration the impact human behavior hason the work effort. EA should be more holistic and much broader in scope and that it include the technical, business, solution architecture as well the impact of technology on both stakeholder and organization behavior.

In the next section, the recursive nature of EA design is listed as a process to be considered and explored in more depth in EA development. We can demonstrate the importance of the recursive nature of EA design if we consider the elicitation of stakeholder input (specifications) as a critical, vital component of EA. The product from the elicitation process must detail the requisite technical functional and nonfunctional specifications and requirements (simply specifications) for organizational IT systems and subsystems. The specifications produced provide and are the foundation for the quantitative and qualitative facets of the EA including 
implementation, performance, reliability, security, maintainability, governance, and compliance with business strategy. Failure to correctly capture, document, and communicate these specifications back to the stakeholders to verify correctness, often leads to and can result in failed EA design and/or implementation, simply stated "poor architecture".

The specification elicitation process as described is iterative, including modeling of the specification, verification of the specification and written acceptance by the stakeholder (redoing the specification if it is invalid) while keeping the effects of new technology in-line with stakeholder capacity for change. In effect, conforming to the recursive behavior described in Giddens' Theory of Structuration [7]. This requires the EIA to maintain a perspective remembering that stakeholder capacity for change is affected by several factors: their past and present organizational experiences, organizational behavior, and what they might perceive and expect in the future. Failure to capture contextual variations in stakeholder perceptions within and across departments within an organization, coupled with failure to communicate and define valid, objective, uniform work flows, processes and information needs can adversely affect EA design.

The regimen imposed by existing EAFS often inhibits innovative, creative problem-solving by limiting stakeholder input typically due to existing organizational rules, procedures, and assumptions [14]. Though the EAFs each provide a mechanism of documenting the EA, there are little or no provisions for incorporating patterns of stakeholder and organizational relationships (i.e., communication) which is essential to monitor and manage human behavior. Communication mechanisms allow management to exercise EA governance and to inform stakeholders of subsequent organizational change caused by the EA. Simply stated, people who will be affected by IT change are unlikely to accept and be impressed by the change if the change is not communicated in a timely effective manner. Therefore, the EA should be predicated upon a communications path, both vertical and horizontal, that recognizes human behavior, the impact of technology, and the actions and relationships of those entities on and within the social structure of the organization. The communications path should foster and encourage a participative EA design that encourages "ownership" by EA stakeholders. In effect, a sociocommunicative path enhanced with a feedback mechanism that provides a means of maintaining a state of homeostasis offering a channel for stakeholders to exchange ideas, knowhow and knowledge benefitting the organization.

\section{Structuration and Information Technology}

Contemporary EA design pervades the IT world driven by the desire to align business strategy with IT resources with the intent of providing a more efficient and effective organizational environment [14]. The current approach to EA design follows traditional software engineering practices that employ a variety of computer science theories and "computeroriented functions" that depends heavily on the elicitation of IT specifications based on explicit and tacit organizational knowledge gleaned from stakeholders. These specifications form the foundation for EA which are then transformed into both functional and non-functional design requirements [14][18].

The typical process used by EIAs to progress EA design consists of historical technology-oriented tools/frameworks that include various techniques (methods), procedures and processes, and paradigms designed to achieve and formulate a desired technological solution [18]. However, these technology-oriented tools/frameworks are deficient and fail to recognize and consider the impact of new or enhanced technology on human behavior which can and may significantly affect the success or failure of EA design [16]. This is especially true in the elicitation of "tacit" stakeholder knowledge, that undocumented knowledge known only to a specific individual or group of individuals that may or may not intentionally withhold that information from the EA, which, if not taken into design consideration, can lead to EAs that are only partially implemented or, in some instances, completely abandoned [6][17]

Users of technology (i.e., stakeholders) have historically accepted the infusion of technology (e.g., hardware, software, and processes) solutions into their daily lives without question. That is no longer true today. Stakeholders frequently question, either covertly or overtly, new or enhanced technology in relation to how it might affect the environment in which they function [16]. This change in the human behavior therefore poses a topic of high relevance to EA management and in how EIAs do their work given that new or enhanced technology can be accepted, rejected, or modified to fit the roles desired by stakeholders [16]. It also raises questions about how EIAs must evaluate the quality of the elicited specifications and how the unanticipated use of technology might affect EA design throughout the EA design effort[16]. Stakeholder use of technology therefore manifests itself in two ways:

- How the solution may be either abused or used properly

- Designing the solution such that it identifies and handles abuse.

If we consider the elicitation of stakeholder specifications and subsequent EA requirements as knowledge creation, simply organizational knowledge held by stakeholders, as the intersection and interaction of technology and stakeholder behavior, then how stakeholders create, process, and provide that knowledge as input to the EA becomes a recursive process directly related to organizational behavior and environment. This knowledge is then a dynamic recursive reconceptualization of organizational knowledge creation that 
directly affects the quality of EA design specifications while providing a paradigm for effective EA.

Organizational behavior is based on a set of standardized rules, procedures, processes, and systems (collectively referred to as rules) [11][12]. These rules constitute a set of coordinated and controlled activities with institutional work (i.e., output) produced from complex networks of technical relations that span organizational boundaries [16]. The rules and activities then become an integral part of prevailing social behavior within the organization and are subsequently built into the society as reciprocated interpretations [3][7]. However, conformity to these rules and activities is often problematic as it conflicts with organizational efficiency by undermining, constraining, and inhibiting organizational flexibility. This rule-based environment inhibits and limits a dynamic view of the organization and discourages participation and, in genreal, is not conducive for EA stakeholders to be innovative, creative, and therefore more receptive to change [7][16]. Our approach considers the recursive nature of people and organizations postulated by Giddens' Theory of Structuration [7] as a theoretical lens to align EA and IT with a socio-communicative framework that takes into account both stakeholder and organizational behavior.

In the Theory of Structuration, the duality of structure, agency, transformation, the relationship between agency (human actors/agents - stakeholders) and structure (systems/organizations/society), are considered in the following contexts:

- How are the actions of individual agents related to the structural features of organizations/society?

- How do individual agents act on a day-to-day basis?

- How are individual actions reproduced?

The theory posits that structure exists only in and through the activities of human agents and gives form to social life but that that form is itself not the shape of it. Giddens' agency does not refer to people's intentions in doing things but focuses on the behavioral patterns exhibited by people's actions. The duality of structure suggests a social structure consisting of rules and resources with rules being applied to govern and regulate social life and resources including both human and non-human elements that can be transformed into power.

The Theory of Structuration examines human actors/agents actions and structures (organization/systems) and is concerned with reworking conceptions of human being and human doing, social reproduction and social transformation. It is based on the premise that human agents or actors operate at both a conscious and unconscious level which constitutes day-to-day behavior and that the routine (i.e., whatever is done habitually) is a basic element of day-to-day social activity (i.e., the recursive and reproductive nature of social life) [7]. It articulates a process-oriented theory that treats structure (institutions) as both a product of, and a constraint on, human action, the result of what actors are able to say about the conditions of their actions and that of others. These discursive phenomena can be detrimental in that human agents/actors may limit or completely avoid to disclose or occlude what they know about what they do and what they say about it. The actions may be intentional or unintentional, conscious or unconscious. Giddens states that all human beings are knowledgeable knowing a great deal about the conditions and consequences of what they do [7]. The knowledgeof human actors is bounded by unconscious action on one hand, and unacknowledged conditions and/or unintended consequences of action on the other. All factors which may affect the veracity of those specifications and requirements needed for quality EA design.

Until recently, the Theory of Structuration, has been a theory based on the social sciences, human action, and organizational structure paying little attention to IT. However, the application of the Theory of Structuration to IT lends itself as a design tool for EIAs to better understand stakeholder behavior and the conceptual impact on organizational behavior assisting in formulating, designing, and implementing EA technology.

One noted advocate for using the Theory of Structuration in IT development and deployment, proposed the Structurational Model of Technology (SMT), as a means to understand how technology affects organizations and vice versa [16]. This approach centers on two concepts - the Duality of Technology and the Interpretive Flexibility of technology [16]. The former posits that the socially created view and the objective view of technology is not exclusive but intertwined and are differentiated because of the temporal distance between the creation of technology and usage of the same. Interpretive Flexibility on the other hand defines the degree to which users of a technology are engaged in how it is built and used (physically and/or socially).

From an organizational perspective, institutional properties and practices influence human and consequentially organizational behavior in their respective interaction with technology. Conflicts, tension, and resistance to change can occur as a result of knowledgeable action on the part of human actors. Human actors use technology including: professional norms; rules of use - design standards and available resources (time, money and skills), which acts upon the institutional structure of an organization. The consequence of the institutional interaction with technology manifests itself by impacting the institutional properties of an organization through reinforcing (sustaining) or transforming (changing) structures of signification, domination and legitimization that characterize the institutional realm.

In summary, the theoretical premise of the Theory of Structuration [7] and SMT [16] acknowledges that 
organizational structures, technology and human action are not distinct and disjoint activities/entities but intertwined such that each is continually reinforced and transformed by the other (a recursive process). It considers human behavior as a product of the environment in which they perform and that they are skilled, innovative, and creative performers that carry and create scripts and develop roles that fit the environment and organization in which they function.

Taken together, the Theory of Structuration and the Structuration Model of Technology highlight those factors which may affect the veracity of those specifications and requirements needed for quality EA design. A logical conclusion can therefore be made that an initiative such as the formulation of enterprise architecture remains incomplete if it does not explicitly take into account human action and organizational behavior from which to derive a dynamic theory of social and institutional order. A solution to this problem can be found in the following section where a where a communications path/channel is established and maintained that encourages knowledge sharing among stakeholders to better ensure and manage EA design.

\section{A Socio-Communicative Approach to Enterprise Architecture Design}

Many of the emergent causal processes that lead to EA failure has been well documented with those factors of significance attributed to EA failure detailed in our earlier paper [13]. The challenge facing an EIA and the organization is how to avoid these factors and prevent EA failure. The answer to this challenge might lie in the ubiquitous nature of communication, its relevance to human behavior, and its implementation as a modifier for human behavior.

If we consider human behavior from a historical perspective, social theorists posit that organizational behavior directly reflects the attitudes and behavior of top management through all layers of the organization [7][11][12]. In concert with this hypothesis, the addition of technology significantly influences human behavior and the attitudes, feedback loops, and selection mechanisms that typically are used in the EA [16][19]. These then form the basis for an analytical approach wherein socio-communicative processes provide a more meaningful and cogent solution to many EA failure factors.

One of the lead factors and often cited as the major contributor and influence for EA failure, lack of or poor leadership, frequently focuses on the role played by the EIA [9]. However, the manner in which the EA is designed and implemented typically poses at least one counter-productive issue that can be easily mitigated and managed by the EIA: poor communication. In most failed EAs, "poor architecture" is most often attributed as the leading factor for the failure. Upon investigation, the design specifications are identified as the major cause for the failure [4][17]. A correlation between the elicitation of stakeholder specifications, understanding of those specifications, and subsequent transformation of those specifications into EA design requirements can easily be drawn upon review and analysis of the root-cause of failure. However, the problem may go far beyond just poor specifications and requirements.

In most organizations, people behave as the group behaves and tend to use technology in ways in which they are both familiar and understand [3][16]. In addition, their actions and motivations typically are influenced and driven by group behavior [8]. Our goal is to establish an environment where human agents/actors (stakeholders) are willing to share explicit, commonly known and perhaps even documented, knowledge and, more importantly as it may be that needed to ensure EA success, that "tacit" knowledge of what and how they really perform their day-to-day activities. In effect, an environment as stated above that promotes, encourages, and fosters user "ownership" of the EA.

The challenge associated with this goal requires that the EA environment transcend technical and business issues and become more focused on the recursive nature and human aspects of the EA. Human behavior then becomes a prime concern of EA design with communication (the exchanging of information, messages, ideas, opinions, and explicit and tacit knowledge) the motivational mechanism used in clarifying otherwise ambiguous goals and objectives and solving complex IS issues and concerns. This aspect of EA then is just as or more important than any technical and/or business acumen. As the mechanism to progress EA design, communication is an effective learnable skill. To illustrate the communications issue, organizations are faced with three behavioral concerns:

- The introduction of new technology into organizations

- Changes in human behavior resulting from new technology

- Resistance to change

Considering each of these factors, people occasionally resist new technology and change for legitimate reasons:

- A fear of job loss and job security

- A perception of loss of status and responsibility within the organization

- A need to learn new procedures and processes

- A feeling the employer no longer cares about the employee

We propose an alternative solution that puts in place a communications mechanism where an environment exists that fosters and encourages:

- Sharing of ideas that facilitate decision-making and problem-solving 
- Explaining decisions and providing an opportunity for clarification

- Sharing responsibility for decision-making and implementation

- Providing specific instructions and closely supervising and rewarding performance (governance)

Our approach establishes, as a first step, EA governance at the beginning of EA design and defines how it will be documented, continued, and maintained throughout the EA life-cycle. The second step requires top-level management to publish a description of what EA is and describing its purpose, the reasons for, and the role EA has within the organization detailing an initial list of EA goals and objectives including the magnitude, scope, and boundaries for the EA. The third step defines and establishes the communications process describing the:

- Format for all EA design and implementation correspondence such as review meetings and the collection of EA specifications

- Mechanism for administering and managing the EA governance

- Procedure for providing input to the EA design for suggesting innovative and creative ideas, opinions, and endogenous and exogenous factors that might influence the decision-making process

- Structure of the communications path including who is to receive copies of EA related correspondence such as news bulletins, interview, and EA related information.

The goal of this proposed framework is to create an architecture that provides best chance for success as well as the most adaptable, practical solution for the future and which aligns strategic business with IT plans.

\section{Discussion, Concluding Remarks, and Future Directions}

Systems of coordinated and controlled activities result from work embedded in complex networks of technology-centric relations and boundary-spanning exchanges. Existing EAFs do not address the behavioral aspects of humans and the organization resulting from technology. The Theory of Structuration provides a means of understanding human behavior and its relationship to organizational change [7]. $S M T$, on the other hand, addresses the effects of technology on human behavior [16]. Taken together, they conceptualize the unique opportunities for an EIA to implement an EA.

This paper explores the possibilities extant and the potential contribution marrying the Theory of Structuration with SMT in an EA can make towards improving the EAF process. It further initiates the clarification of an ontology and paradigm describing an architectural framework that encourages an environment that fosters stakeholder participation and collaboration in the EA design process and a forum for information-sharing. It allows human agents/actors to communicate whenever and however they need to in order to solve problems and exchange knowhow and knowledge.

The possibility and prospect of a successful EA becomes realizable if an enhanced participative working environment is encouraged and made a part of the design and implementation process that welcomes new EA technology and avoids it being perceived as a risk or threat to stakeholder well-being. At the same time, it provides the mechanism for EA governance documenting the EA from inception throughout its life-cycle. Future work expands the framework clarification above in more depth and detail. The goal of this work is to provide a communications process (a framework in its own right) that can be used in conjunction with existing EAF methodologies with the potential it offers for removing many of the behavioral obstacles which inhibit EA deployment.

\section{References:}

[1] No author listed, The DoD Architecture Framework Version 2.0DoD Deputy Chief Innfromation Officer, Department of Defense, April, 2010.

[2] Chief Information Officers Council, Federal Enterprise Architecture Framework, CIO Council, Version 1.1, August 5, 1999.

[3] E. Davidson, Technological Frames Perspective on Information Technology and Organizational Change, The Journal of Applied Behavioral Science, 2006 : 42: 23

[4] C. Ferreira and J. Cohen. "Agile Systems Development and Stakeholder Satisfaction: A South African Empirical Study," Proceedings 2008 Conference of South African Institute Computer Scientists and Information Technologists on IT Research in Developing Countries, pp. 48-55, 2008.

[5] J. Gido and J. Clements, Successful Project Management, South Western CengAGE Learning, $4^{\text {th }}$ Edition, 2009.

[6] R. Gauld. "Public Sector Information System Failures: Lessons from a New Zealand Hospital Organization," Government Information Quarterly, 24(1):102-114, 2007.

[7] A. Giddens. The Constitution of Society: Outline of the Theory of Structuration, University of California Press, 1984.

[8] P. Hersey abd K. H. Blanchard, Nabagement of Organizational Behavio: Utilizing Human Resources, 5th Edition, Prentice-Hallm Inc., Englewood Cliffs, NJ, 1988

[9] R. Lewin and B. Regine. "Enterprise Architecture, People, Process, Business, Technology," Institute for Enterprise Architecture Developments [Online], Available: $\quad$ http://www.enterprisearchitecture.info/Images/Extended\%20Enterprise/Extende d\%20Enterprise \%20Architecture 3.html. 
[10] A. Lindstrom, et al, A Method to Assess the Enterpriesewide IT Resource for Performance and Investment Justification, Dept of Industrial Information Systems and control Systems, Royal Institute of Technology, KTH, SB-100, 44 Sto0ckholm. Sweden, 2005

[11] A. Maslow. Motivation and Personality, Harper Collins, 1987.

[12] D. McGregor. The Human Side of Enterprise, McGrawHill, 1960.

[13] D. M. Mezzanotte, Sr., J. Dehlinger, and S. Chakraborty, On Applying the Theory $f$ Structuration in Enterprise Architecture Design, IEEE/ACIS, August, 2010.

[14] D. Minoli. Enterprise Architecture A to Z, CRC Press, New York, 2008.

[15] The Open Group, TOGAF Version 9, The Open Group, 2009.
[16] W. Orlikowski and D. Robey, Information Technology and the Structureing of Organizations, The Institute of Management Science, 1991.

[17] S. Roeleven, Sven and J. Broer. "Why Two Thirds of Enterprise Architecture Projects Fail," ARIS Expert Paper [Online], Available: http://www.ids-scheer.com/set/ 6473/EA_-_Roeleven_Broer_-_Enterprise_Architecture _Projects_Fail_-_AEP_en.pdf.

[18] N. Rozanski and E. Woods, Software Systems Architecture, Addison-Wesley Professional, 2006.

[19] C. Seaman, Qualitative Methods in Empirical Studies of Software Engineering, IEEECS, Log Number 109541, June, 1998.

[20] J. Zachman, Concepts of the Frameword for Enterprise Architecture, Information Engineering Services, Pty, Ltd., No Date. 